\title{
PRURITUS IN SYSTEMIC SCLEROSIS, A THERAPEUTIC CHALLENGE: A CASE REPORT
}

\begin{abstract}
Gabriela Almeida Barbosa ${ }^{1, \star}$, Robson Antônio Gonçalves ${ }^{1}$, Valéria Bezerra da Silva ${ }^{1}$, Tamara Santos Meloํ․ Maria Roberta Melo Pereira Soares ${ }^{1}$, Ana Karla Guedes de Melo ${ }^{1}$, Danielle Christinne Soares Egypto ${ }^{1}$, Sandra Rejane Cabral Batista ${ }^{1}$, Alessandra de Sousa Braz ${ }^{1}$, Eutilia Andrade Medeiros Freire ${ }^{1}$
\end{abstract}

1.Universidade Federal da Paraíba, João Pessoa (PB), Brazil.

*Corresponding author: gabrielaalmeidamed@gmail.com

\section{BACKGROUND}

Pruritus in systemic sclerosis (SSc) is present in about $40 \%$ of patients, with a significant impact on quality of life (QOL) and uncertain pathophysiology. It is associated with worsening of the skin and gastrointestinal involvement and is most commonly seen on the head, back and extremities. Despite the high frequency, its management remains uncertain and based on the use of symptomatic.

\section{CASE REPORT}

Woman, 61-year-old, diagnosed with systemic lupus erythematosus (SLE) 20 years ago, polyarthritis, serositis, alopecia, oral ulcers and nuclear speckled 1:640 ANA (SLICC criteria, 2012), in irregular follow-up and use of azathioprine $2 \mathrm{mg} / \mathrm{kg} / \mathrm{day}$. It evolved 5 years ago with generalized pruritus, unintentional weight loss and Raynaud's phenomenon (RP), with significant impact on QOL and progressive worsening for 6 months. Admitted to university hospital for investigation. On admission, she presented generalized pruritus without skin lesions (grade 9/10), telangiectasias in the face and mucosa and sclerodactyly. Complementary exams showed: mammography, upper digestive endoscopy, colonoscopy, thyroid ultrasonography (USG), transvaginal pelvic USG and abdominal computed tomography (CT) without alterations; serology for hepatitis B and C, syphilis and HIV negative; chest CT with nonspecific interstitial lung disease (NSIP), presence of ground glass in approximately $30 \%$ of the parenchyma, spirometry not available; anti-RNP positive at low titers (1:40), anti-Ro, anti-La, anti-Jo-1, anti-Scl-70 negative. Given the presence of sclerodactyly, RP, telangiectasias and interstitial lung disease, she met ACR/EULAR 2013 criteria for SSc. Treatment was initiated with hydroxychloroquine $400 \mathrm{mg} / \mathrm{day}$, cyclophosphamide (CYC) $1 \mathrm{~g} /$ month for pulmonary involvement, nifedipine $20 \mathrm{mg} /$ day and, for the treatment of pruritus, loratadine $10 \mathrm{mg} /$ day, dexchlorpheniramine $4 \mathrm{mg} /$ day, prednisone $15 \mathrm{mg} /$ day and amitriptyline $50 \mathrm{mg} /$ day. After starting CYC, the itching improved significantly (score 2/10), with progressive weaning from antihistamines and prednisone.

\section{CONCLUSION}

The management of pruritus in SSc remains a challenge in clinical practice. Clinical trials that seek to find more effective therapeutic alternatives for the treatment of pruritus should be encouraged, given the high impact on the QOL of these patients. The reported case shows a significant pruritus response with the use of $\mathrm{CYC}$, which may corroborate the hypothesis of an autoimmune component in the pathophysiology of this symptom.

\section{KEYWORDS}

Pruritus, Autoimmunity, Systemic sclerosis. 